\title{
Valoración de los niveles de calidad del aire de interiores en espacios de institución de educación superior
}

\section{Assessing Indoor Air Quality Levels in Higher Education Institutions}

Mónica V. Sandoval', Nicolás M. Solano'1, Laura K. Gualdrón', Juliana C. Meneses'

\section{Resumen}

La calidad del aire de interiores (CAI) es determinante cuando se trata de la salud respiratoria y del confort de las personas. El objetivo de esta investigación se centró en evaluar el nivel de calidad del aire interior (CAI) a través de encuestas de percepción efectuadas a usuarios y mediciones de temperatura, humedad relativa, PM10, PM2.5, $\mathrm{CO}_{2}, \mathrm{CO}$ y $\mathrm{HCHO}$ en aulas de clase de una institución de educación superior. Las encuestas realizadas a los ocupantes mostraron que la principal inconformidad percibida fue la alta temperatura del lugar, la cual osciló entre $19,9^{\circ} \mathrm{C}$ y $29^{\circ} \mathrm{C}$. La humedad relativa presentó valores entre $64,3 \%$ y $93 \%$, superando el rango sugerido (30\% - 60\%). La concentración máxima de PM10 $\left(41 \mu \mathrm{g} / \mathrm{m}^{3}\right)$ no superó el límite establecido por la Ashrae $\left(50 \mu \mathrm{g} / \mathrm{m}^{3}\right)$, mientras que la concentración máxima de PM2.5 $\left(21 \mu \mathrm{g} / \mathrm{m}^{3}\right)$ sobrepasó el límite sugerido $\left(15 \mu \mathrm{g} / \mathrm{m}^{3}\right)$. Las concentraciones de los gases $\mathrm{CO}_{2}$ (998 ppm), $\mathrm{CO}$ (21 ppm), y HCHO $\left(0,04 \mathrm{mg} / \mathrm{m}^{3}\right)$ mostraron siempre valores inferiores a los límites estipulados por las diferentes entidades regulatorias.

Palabras clave: calidad del aire de interiores, síntomas, material particulado, monóxido de carbono, dióxido de carbono.

1 Grupo de Investigación en Ingeniería Ambiental y Recursos Naturales (GINAM), Facultad de Ingeniería Ambiental, Universidad Santo Tomás, seccional Bucaramanga, Cra. 27 №. 180-395, Floridablanca, Santander. Correo: monica.sandovaldustabuca.edu.co 


\section{Abstract}

Indoor Air Quality (IAC) is critical when it comes to people's respiratory health and comfort. This research assesses IAC through user perception surveys and measurements of temperature, relative humidity, $\mathrm{PM} 10, \mathrm{PM} 2.5, \mathrm{CO} 2, \mathrm{CO}$, and $\mathrm{HCHO}$ in classrooms of a higher education institution. The surveys showed that the primary complaint was the high temperature of the place, which ranged between 19.9 and $29{ }^{\circ} \mathrm{C}$. Relative humidity ranged between 64.3 and $93 \%$, exceeding the suggested range $(30-60 \%)$. The maximum concentration of PM10 $(41 \mu \mathrm{g} / \mathrm{m} 3)$ did not exceed the limit established by the ASHRAE $(50 \mu \mathrm{g} / \mathrm{m} 3)$, while the maximum concentration of PM2.5 $(21 \mu \mathrm{g} / \mathrm{m} 3)$ surpassed the suggested limit $(15 \mu \mathrm{g} / \mathrm{m} 3)$. The concentrations of CO2 (998 ppm), CO (21 ppm), and $\mathrm{HCHO}(0.04 \mathrm{mg} / \mathrm{m} 3)$ were consistently lower than the limits stipulated by different regulators.

Keywords: Indoor air quality, symptoms, particulate matter, carbon monoxide, carbon dioxide.

\section{Introducción}

En los últimos años, los estudios sobre contaminación del aire de interiores (CAI) han comenzado a recibir atención tanto en la sociedad como en la comunidad científica, especialmente porque la calidad del aire en áreas de estudio o de trabajo tiene efectos adversos no solo en la salud, sino también en la memoria, la concentración y la comodidad (Argunhan y Avci, 2018). De acuerdo con la Organización Mundial de la Salud (OMS), las personas que viven en zonas urbanas pasan gran parte de su tiempo $(\sim 90 \%)$ en recintos cerrados, por lo cual, los niveles de concentración de contaminantes en estas zonas son un factor determinante para la salud y el bienestar (WHO, 2006). De hecho, algunos estudios han demostrado que los niveles de contaminantes en el interior son a menudo mucho más altos que los encontrados típicamente en el exterior. A nivel mundial, se calculó que de los 56,4 millones de defunciones registradas para el 2016, el 12,3\% fueron causadas por la enfermedad pulmonar obstructiva crónica (EPOC), cáncer de pulmón, tráquea o bronquios, e infecciones (transmisibles) de las vías respiratorias (WHO, 2000). 
Asimismo, la OMS en el 2018 reportó que más del $50 \%$ de las muertes por neumonía en niños $(<5$ años) fueron causadas por partículas inhaladas en espacios cerrados (OMS, 2018). En Colombia, por ejemplo, estas enfermedades están incluidas dentro de las diez principales causas de muerte, cobrando aproximadamente 26268 vidas al año (defunciones ocurridas entre el $1^{\circ}$ de enero y el 31 de diciembre del 2019) (DANE, 2020).

El aire limpio dentro de espacios cerrados, en los cuales las personas pasan gran parte de su vida, es un determinante esencial de una vida sana. El material particulado y las sustancias peligrosas emitidas, ya sea por actividades humanas en el interior de los edificios o por el transporte de los contaminantes desde el exterior, ha empezado a evidenciar en la población, tanto problemas de salud como dificultades en la percepción de un ambiente confortable de estudio o trabajo (Penney et al., 2010). A nivel nacional, existen pocas investigaciones que evidencien las condiciones ambientales de lugares cerrados con actividades no industriales. Una primera aproximación al mejoramiento de la calidad de vida estaría direccionada a impulsar estrategias para reducir el riesgo y la inconformidad (Instituto Nacional de Seguridad e Higiene en el Trabajo, 2015; Kirchner et al., 2002).
El Síndrome del Edificio Enfermo (SEE) es el conjunto de enfermedades originadas o estimuladas por la contaminación del aire en lugares cerrados, que produce, al menos, $20 \%$ en los ocupantes, síntomas tales como sequedad e irritación de las vías respiratorias, irritación de piel, ojos, nariz y garganta, eritemas, dolor de cabeza, fatiga mental, alergias, resfriados persistentes e hipersensibilidades inespecíficas, sin que sus causas estén perfectamente definidas. Es característico que los síntomas desaparezcan al abandonar el lugar (Mentese et al., 2020; Ministerio de Trabajo y Asuntos Sociales España, 2003).

Esta investigación tiene como objetivo indagar la CAI en un aula de clase mediante encuestas y mediciones in situ de temperatura, humedad relativa, PM10, PM2.5, CO2, CO y formaldehído. Asimismo, se exponen las sensaciones y síntomas perceptibles por los usuarios del espacio, y la relación de los mismos con los parámetros de confort y calidad del aire.

\section{Materiales y métodos}

Este estudio se realizó en un aula de clase ubicada en el cuarto piso de un edificio con 18 años de antigüedad, localizado en una institución de educación superior de 
la ciudad de Bucaramanga, Colombia. El aula posee un área de $15 \mathrm{~m}^{2}$, una puerta de madera, una ventana de vidrio de 7,5 $\mathrm{m} 2$, tres lámparas de luz fluorescente de $2 \mathrm{~m}$ de largo, un escritorio de madera y aproximadamente 22 sillas de tubería redonda y polipropileno. En cuanto a la ventilación, el aula no cuenta con ventiladores o sistema de aire acondicionado.

Con el fin de evaluar la percepción de la calidad del aire interior y los síntomas experimentados por los usuarios del aula, se realizó una encuesta de confort a 40 personas durante una semana. El cuestionario incluyó diez preguntas que abarcaron información general sobre el tiempo de permanencia en espacios cerrados y conocimientos sobre fuentes de contaminación, además de la percepción de la calidad del aire, confort, y aparición de síntomas cuando se encontraban dentro del lugar bajo estudio (Bright et al., 1992). La cuantificación de los niveles de percepción para las condiciones de confort se hizo con un modelo de respuesta de "muy desagradable", "desagradable", "agradable" y "muy agradable". En el caso de la frecuencia con la cual se experimentaron las sensaciones de confort, se utilizó una escala de "nunca", "algunas veces", "frecuentemente" y "siempre".

El monitoreo de la calidad del aire interior se llevó a cabo durante cinco días, en la franja horaria de 8:00 a.m. a 12:00 m., tiempo en el cual el aula se encontraba ocupada según la programación de clases. La ocupación cambió cada dos horas, teniendo en cuenta el sistema de enseñanza con clases de dos horas de duración. Los parámetros físicos y químicos evaluados fueron temperatura, humedad relativa, PM2.5, PM10, dióxido de carbono (CO2), monóxido de carbono (CO) y formaldehído (HCHO). Un contador de partículas portátil PCERCM11 (PCE Instruments) se utilizó para la medición ambiental orientativa/ detección de temperatura (resolución $\left.0,1^{\circ} \mathrm{C}\right)$, humedad relativa $(0,1 \% \mathrm{rH})$, partículas PM2.5 y PM10 (resolución $1 \mu \mathrm{g} / \mathrm{m} 3$ ), CO2 (resolución $1 \mathrm{ppm}$ ) y HCHO (resolución 0,01 mg/m3). La concentración de $\mathrm{CO}$ se determinó usando un medidor de monóxido de carbono modelo CO10 (Extech Instrument), resolución 1 ppm (Extech Instruments, 2020). Los equipos de medición se ubicaron en el centro del aula, colocados en una superficie fija a $1 \mathrm{~m}$ sobre el nivel del piso. Todos los datos se recolectaron cada 5 minutos durante 4 horas.

\section{Resultados y discusión}

El estilo de vida de la población que habita en zonas urbanas conduce a que las personas pasen gran parte de su tiempo en 
espacios cerrados. De acuerdo con los resultados de este estudio, el $60 \%$ del grupo poblacional encuestado pasa entre 7 y 10 horas en diferentes lugares cerrados de la institución educativa, entre ellos, aulas de clase, bibliotecas, cafeterías, oficinas, entre otros (véase Apéndice A). Estas cifras exponen la importancia entre la calidad del aire interior (CAI) y la salud y el bienestar de los ocupantes (SCHER, 2007). La figura 1(a) muestra las sensaciones experimentadas por los ocupantes del aula bajo estudio, respecto a algunos factores que influyen en la CAI, a través de respuestas de agrado o desagrado. Los problemas ambientales que mostraron mayor desagrado fueron la temperatura, el ruido y la ventilación. Para estos parámetros, el $76 \%$ de los encuestados manifestaron una sensación térmica muy desagradable (45\%) o desagradable (31\%), debido a una alta temperatura. Respecto al ruido, el $54 \%$ de la población encuestada manifestó una sensación de desagrado, por exceso de ruido proveniente del exterior. Además, la ventilación insuficiente fue el problema ambiental que mostró mayores molestias o inconformidades (77\%). Otros parámetros como la humedad y la presencia de polvo o suciedad presentaron una distribución similar respecto a las sensaciones de desagrado y agrado. Por ejemplo, para la humedad, el $42 \%$ de la población manifestó desagrado, mientras que el 38\% la percibió agradable. Finalmente, la iluminación del espacio se experimentó como agradable (58\%) o muy agradable (17\%).
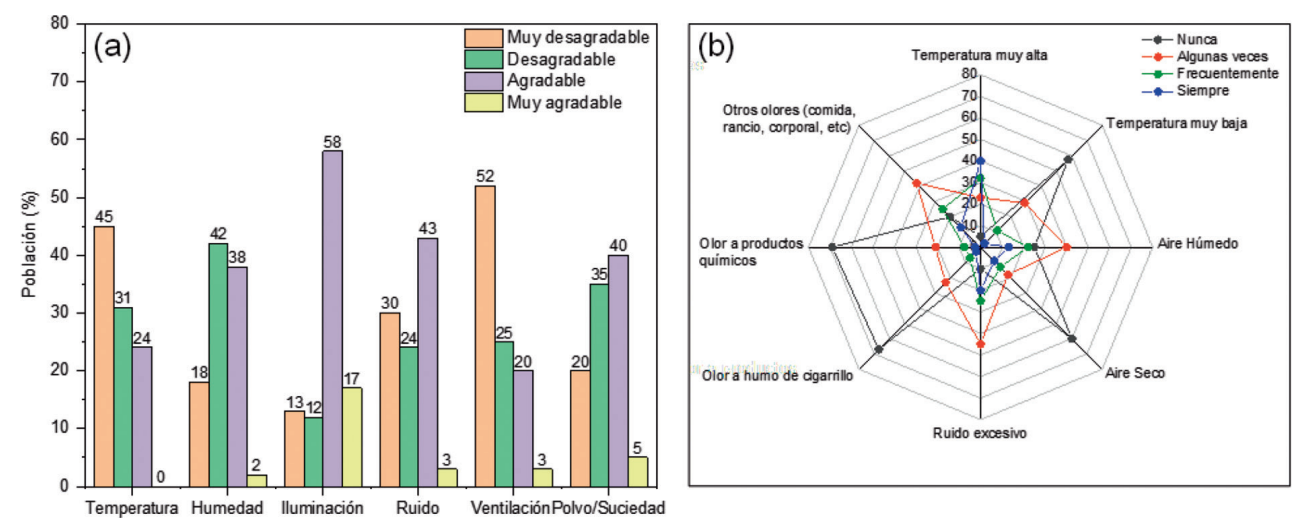

Figura 1. (a) Sensaciones experimentadas por los ocupantes (agradabilidad) y (b) Frecuencia de percepción de parámetros ambientales 
Con el propósito de complementar las respuestas de los encuestados respecto a la calidad del aire dentro del aula, se evaluó la frecuencia con la que los ocupantes percibieron diferentes sensaciones de confort dentro del espacio. Como se muestra en la figura 1(b), la sensación experimentada con mayor frecuencia por la mayoría de los ocupantes fue la temperatura alta, donde el $40 \%$ de la población siempre sintió un ambiente caluroso, debido principalmente a la falta de ventilación artificial o presencia de corriente de aire natural. Otros parámetros como la sensación de aire húmedo, el ruido excesivo y la presencia de olores fueron percibidos solo algunas veces dentro del aula de clase. Por otra parte, los ocupantes nunca detectaron olor a humo de cigarrillo (67\%) o productos químicos (69\%). Asimismo, se consultaron algunos síntomas comunes en personas que trabajan o estudian bajo una rutina establecida. Como resultado, las personas asociaron la aparición de síntomas como dolor de cabeza (55\%) y rinitis, estornudos o secreciones nasales $(50 \%)$ con la excesiva permanencia y desarrollo de actividades en espacios confinados (véase Apéndice B).

Como se observa en la figura 2(a), la temperatura interior osciló entre $19,9{ }^{\circ} \mathrm{C}$ y $29^{\circ} \mathrm{C}$, con un valor promedio de $26,1^{\circ} \mathrm{C}$, el cual está por encima del rango estándar $\left(20^{\circ} \mathrm{C}\right.$ a $\left.25^{\circ} \mathrm{C}\right)$ especificado para espacios cerrados por la American Society of Heating Refrigerating and Air-Conditioning Engineers (Ashrae) (Ashrae, 2020). La ANSI/Ashrae Standard 55 relaciona la temperatura en edificios condicionados naturalmente con la media mensual de la temperatura exterior. Esta relación está dada por medio de la ecuación (1) para la temperatura de confort (Tconf), donde Tom es la temperatura media mensual al aire exterior (CIBSE, 2013).

Tconf $=0,31$ Tom $+17,8$

Teniendo en cuenta la temperatura promedio mensual de Bucaramanga durante octubre del 2019 (mes en el cual se realizó el estudio), la cual fue de $21,2^{\circ} \mathrm{C}$ (Ideam, 2019), la temperatura de confort sugerida para el aula fue de $24,4^{\circ} \mathrm{C}$. En consecuencia, para evitar la sensación calurosa manifestada por los ocupantes, es recomendable la adición de un sistema de ventilación artificial eficiente que permita disminuir la temperatura del lugar y alcanzar la temperatura de confort recomendada. Con respecto a la humedad relativa (véase Apéndice C), esta fluctuó entre $65 \%$ y $93 \%$ a medida que transcurrió la jornada de la mañana, con un valor promedio semanal de $76,5 \%$. De modo 

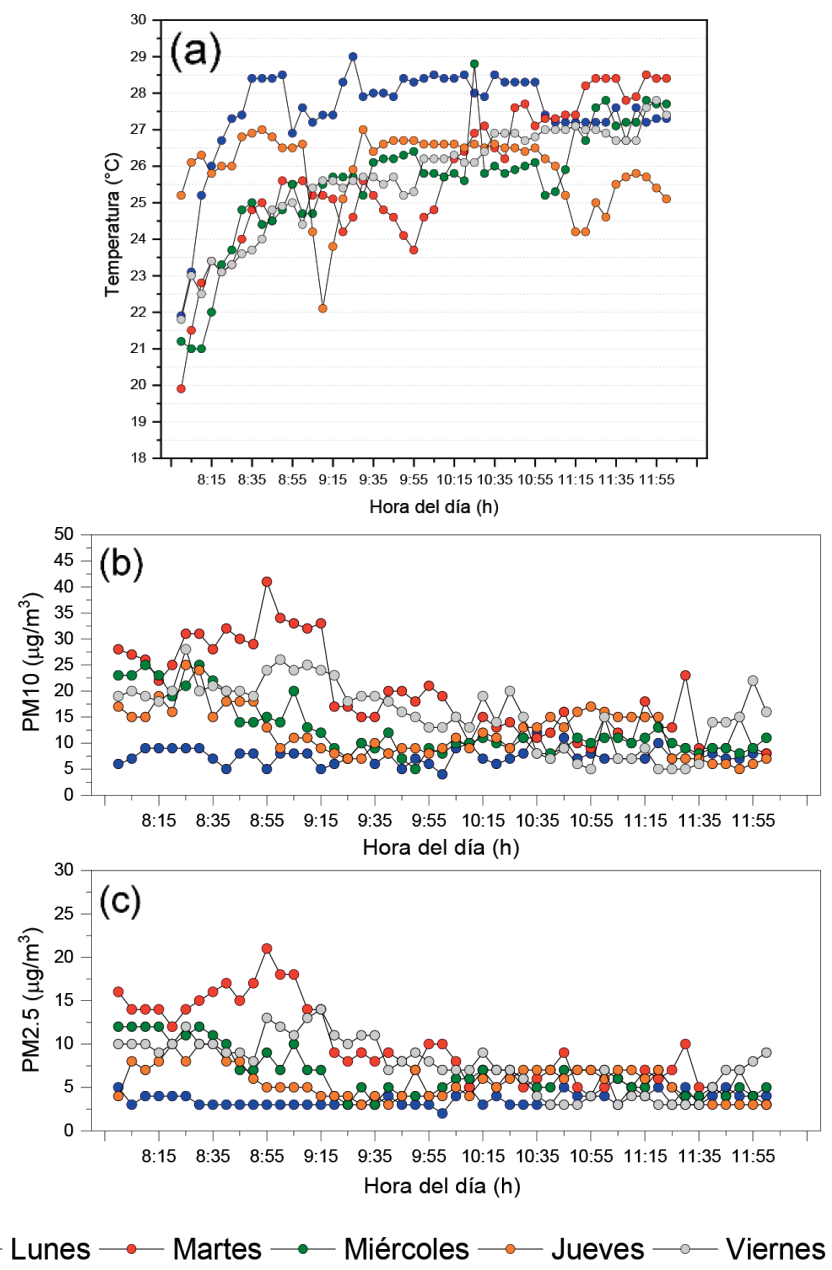

Figura 2. (a) Variación de la temperatura, (b) Variación de la concentración de PM10 y (c) Variación de la concentración de PM2.5 en el aula

que los valores resultantes para el aula excedieron los estándares Ashrae, los cuales refieren un rango de $30 \%$ a $60 \%$. En este caso, es importante controlar la humedad relativa, para minimizar el crecimiento de organismos alergénicos o patógenos (ANSI/Ashrae, 2019).
La concentración de material particulado PM10 (figura 2b) presentó un valor promedio semanal de $13,8 \mu \mathrm{g} / \mathrm{m} 3$. Por otra parte, la concentración de PM2.5 (figuras 2c) mostró un valor promedio de $6,8 \mu \mathrm{g} / \mathrm{m} 3$. El día martes entre las 8:25 y las 9:15 a.m. ambos contaminantes 
presentaron valores elevados, de 30 a $41 \mu \mathrm{g} / \mathrm{m} 3$ para PM10 y de 15 a $21 \mu \mathrm{g} /$ m3 para PM2.5. Durante los días bajo estudio, las concentraciones medidas de material particulado PM10 no superaron el límite establecido por la Ashrae de 50 $\mu \mathrm{g} / \mathrm{m} 3$. Por otra parte, en algunos periodos del día martes las concentraciones de PM2.5 sobrepasaron el límite máximo sugerido de $15 \mu \mathrm{g} / \mathrm{m} 3$ (Ashrae, 2007).

La concentración de $\mathrm{CO} 2$ es un indicador útil de insuficiencia de aire fresco, para el cual la Ashrae sugiere un valor máximo de 1000 ppm, aunque algunas investigaciones han expuesto que concentraciones por encima de $600 \mathrm{ppm}$ generan efectos fisiológicos, lo que conduce a incomodidad e insatisfacción con el ambiente (Bright et al., 1992). Por lo que se refiere a la concentración de $\mathrm{CO} 2$ en el aula bajo estudio, esta presentó un valor promedio semanal de 451 ppm (véase Apéndice D), cumpliendo con los estándares de un espacio confortable. La figura 3 muestra que las concentraciones de $\mathrm{CO} 2$ parecen estar influenciadas fuertemente por la ocupación humana (véase Apéndice D para los días lunes, martes y miércoles). Específicamente, el CO2 empezó a acumularse cuando los ocupantes ingresaron al aula, y disminuyó notoriamente cuando el salón estuvo vacío o con un máximo de tres personas (Wu et al., 2018).
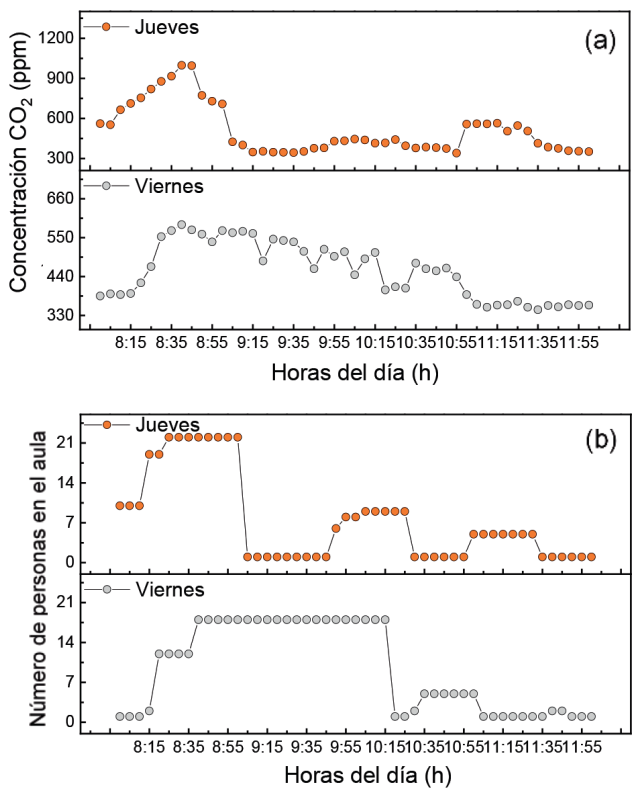

Figura 3. (a) Variación de la concentración de $\mathrm{CO}_{2}$ y (b) Ocupación del aula para los días jueves y viernes

Por otra parte, se encontró que la concentración promedio de monóxido de carbono $\mathrm{CO}$ fue de 11,5 ppm durante el periodo estudiado (véase Apéndice E). Específicamente, los valores más altos (17 a $21 \mathrm{ppm}$ ) se presentaron el día lunes entre las 11:15 a.m. y las 12:00 m. Este comportamiento puntual se debió, posiblemente, al hecho de que durante este periodo se encendió una planta generadora de energía alimentada con combustible, ubicada en la parte exterior (primer piso) del edificio. No obstante, en ningún momento se superó el límite de exposición permisible actual 
de la Administración de Seguridad y Salud Ocupacional (OSHA, por sus siglas en inglés) de $50 \mathrm{ppm}$ con un promedio ponderado de tiempo de 8 horas (TWA en inglés), o el límite de exposición recomendado por el Instituto Nacional para la Seguridad y Salud Ocupacional (NIOSH, por sus siglas en inglés) de 35 ppm (TWA 8 horas) (NIOSH, 1992; OSHA, 2020). Finalmente, la concentración de formaldehído $\mathrm{HCHO}$ siempre se mantuvo muy cercana a cero, presentando un máximo esporádico de $0,04 \mathrm{mg} /$ $\mathrm{m}^{3}$ el día miércoles (véase Apéndice $\mathrm{F}$ ). Este valor fue inferior al máximo permisible de $0,1 \mathrm{mg} / \mathrm{m}^{3}$ estipulado por la Ashrae (Ashrae, 2020).

\section{Conclusiones}

En este trabajo se evaluó la calidad del aire de interiores (CAI) en un aula de clase de una institución de educación superior de la ciudad de Bucaramanga. Las encuestas de percepción mostraron que los parámetros ambientales que generaron mayor desagrado en los ocupantes fueron la alta temperatura (entre $19,9^{\circ} \mathrm{C}$ y $29^{\circ} \mathrm{C}$ ), el exceso de ruido proveniente del exteriory la falta de circulación de aire natural o ventilación artificial. La humedad relativa presentó un valor promedio semanal de $76,5 \%$, el cual excedió los estándares Ashrae (entre 30\% y 60\%).
La concentración máxima de material particulado PM10 $\left(41 \mu \mathrm{g} / \mathrm{m}^{3}\right)$ no sobrepasó el límite establecido por la Ashrae $\left(50 \mu \mathrm{g} / \mathrm{m}^{3}\right)$. Mientras que para el material particulado PM2.5 se presentaron valores altos entre 16 y $21 \mu \mathrm{g} / \mathrm{m}^{3}$, los cuales superan el máximo sugerido de $15 \mu \mathrm{g} / \mathrm{m}^{3}$. La concentración de $\mathrm{CO}_{2}$ fue notoriamente influenciada por la ocupación del espacio, presentando un valor promedio semanal de $451 \mathrm{ppm}$, el cual no superó el valor máximo de 1000 ppm indicado por la Ashrae. Por otra parte, las concentraciones de los gases $\mathrm{CO}$ y HCHO exhibieron esporádicamente algunos valores altos; sin embargo, dentro del rango de tiempo estudiado, las concentraciones de estos gases se encontraron siempre por debajo de los límites máximos mencionados por los diferentes organismos regulatorios (OSHA, NIOSH y Ashrae).

\section{Agradecimientos}

Los autores agradecen a la Universidad Santo Tomás por la financiación realizada a través del proyecto de semilleros de investigación No. 01948263.

\section{Referencias}

ANSI/Ashrae. (2019). Ventilation for acceptable indoor air quality. Standard 62.1-2019. 
Argunhan, Z., \& Avci, A. S. (2018). Statistical evaluation of indoor air quality parameters in classrooms of a university. Advances in Meteorology, 1, 1-10.

Ashrae. (2007). ANSI/Ashrae. Standard 62.12007 - Ventilation for acceptable indoor air quality. American National Standard ANSI 2007: 1-48.

Ashrae. (2020). American Society of Heating, Refrigerating and Air-Conditioning Engineers. https://www.ashrae.org/technical-resources/standar.

Bright, D., Mader, M., Carpenter, D., \& Hermon-Cruz, I. (1992). Guide for indoor air quality surveys. Armstrong Laboratory, Texas, Estados Unidos, 1-53.

CIBSE. (2013). CIBSE TM52. The limits of thermal comfort: Avoiding overheating in european buildings. https://www.cibse.org/ Knowledge/knowledge-items/detail?id=a0q20000008I7f5AAC.

Departamento Administrativo Nacional de Estadística (DANE). (2020). https://www. dane.gov.co/index.php/estadisticas-por-te$\mathrm{ma} / \mathrm{salud} /$ nacimientos-y-defunciones/defunciones-no-fetales/defunciones-no-fetales-2019.

Extech Instruments. (2020). Manual de usuario. http://Www.Extech.Com.Es/Instruments/ Product.Asp?Catid $=7 \&$ prodid $=15$

Ideam. (2019). Boletín Climatológico. Seguimiento mensual de la temperatura media. http://www.ideam.gov.co

Instituto Nacional de Seguridad e Higiene en el Trabajo. (2015). Calidad de ambiente interior en oficinas. http://www.insht.es/ InshtWeb/Contenidos/Documentacion/fi- chas de publicaciones/en catalogo/higiene/ cai en oficinas.pdf.

Kirchner, S. et al. (2002). The french permanent survey on indoor air quality survey design in dwellings and schools. Proceedings: Indoor Air, 1, 449-454.

Mentese, S. et al. (2020). A long-term multi-parametric monitoring study: Indoor Air Quality (IAQ) and the sources of the pollutants, prevalence of sick building syndrome (SBS) symptoms, and respiratory health indicators. Atmospheric Pollution Research Journal Pre-proof. https://doi.org/10.1016/j.apr.2020.07.016.

Ministerio de Trabajo y Asuntos Sociales España. (2003). NTP 289: Sindrome del edificio enfermo: factores de riesgo. España. http://www.ugt-cat.net/subdominis/ajllei$\mathrm{da} /$ images/stories/documents/salutlaboral/ lipoatrofia_semicircular/ntp_289_sindrome_del_edificio_enfermo_factores_de riesgo.pdf.

NIOSH. (1992). Recommendations for occupational safety and health: Compendium of policy documents and statements. Cincinnati, OH: U.S. Department of Health and Human Services, Public Health Service, Centers for Disease Control and Prevention. Ohio, Estados Unidos.

Organización Mundial de la Salud (OMS). (2018). Contaminación del aire de interiores y salud. https://www.who.int/es/newsroom/fact-sheets/detail/household-air-pollution-and-health.

OSHA. (2020). Occupational Safety and Health Administration. https://www.osha.gov/ 
Penney, D. et al. (2010). Guidelines for indoor air quality. WHO guidelines, 9, 454-460.

SCHER. (2007). Scientific Committee on Health and Environmental. European Commission: Health \& Consumer Protection Directorate - General opinion on risk assessment on indoor air quality. http:// ec.europa.eu/health/ph_risk/risk_en.htm.

WHO. (2006). Development of WHO guidelines for indoor air quality - Report on a working group meeting. World Health Organization Regional Office for Europe, 1-27. http://www.euro.who.int/_data/ assets/pdf_file/0007/78613/AIQIAQ_mtgrep_Bonn_Oct06.pdf.
WHO. (2000). Environmental science and pollution research air quality guidelines for Europe. World Health Organization Regional Office for Europe, 1-68. http://link. springer.com/10.1007/BF02986808\%5Cnhttp://www.springerlink.com/index/10.1007/BF02986808.

Wu, Y., Lu, Y., \& Chou, D-Ch. (2018). Indoor air quality investigation of a university library based on field measurement and questionnaire survey. International Journal of Low-Carbon Technologies, 13(2), 148-160. 


\section{Apéndice A}

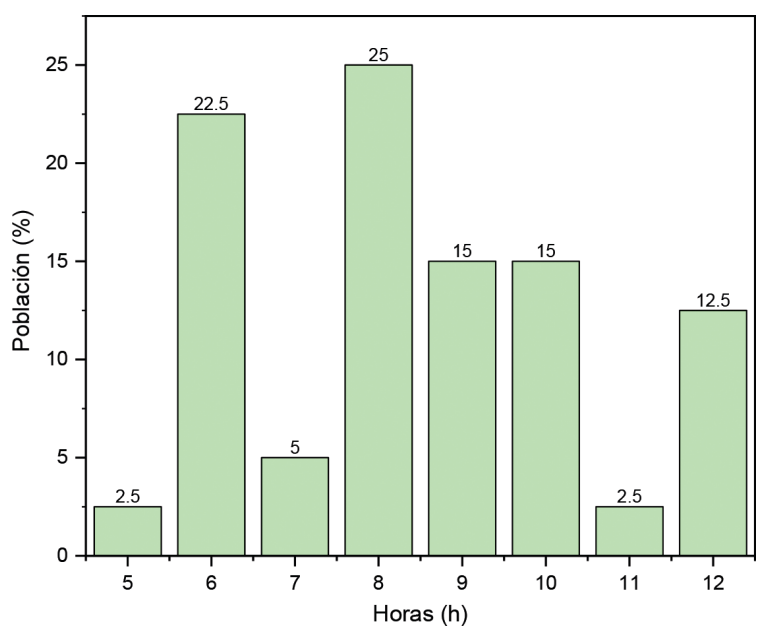

Figura A1. Tiempo de permanencia

de los encuestados en lugares cerrados dentro de la universidad

\section{Apéndice B}

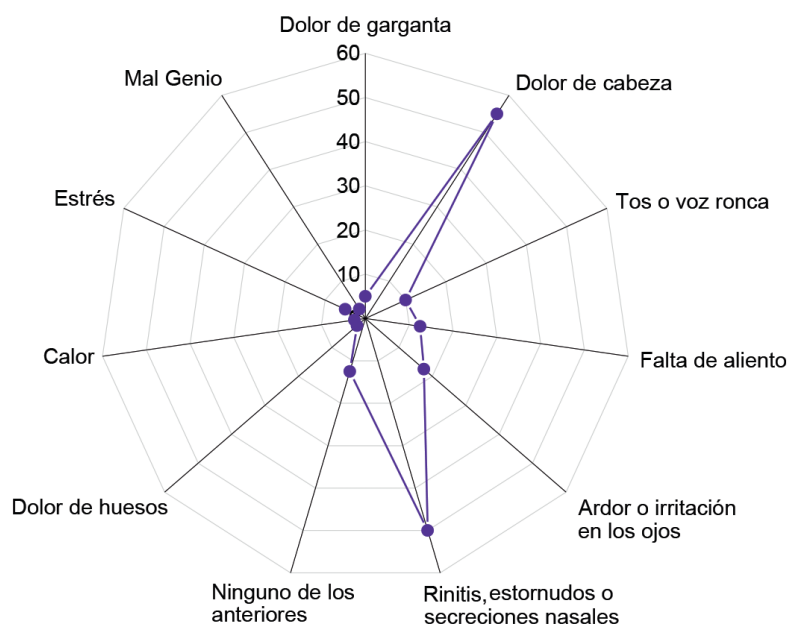

Figura B1. Síntomas experimentados por los ocupantes del aula 


\section{Apéndice C}

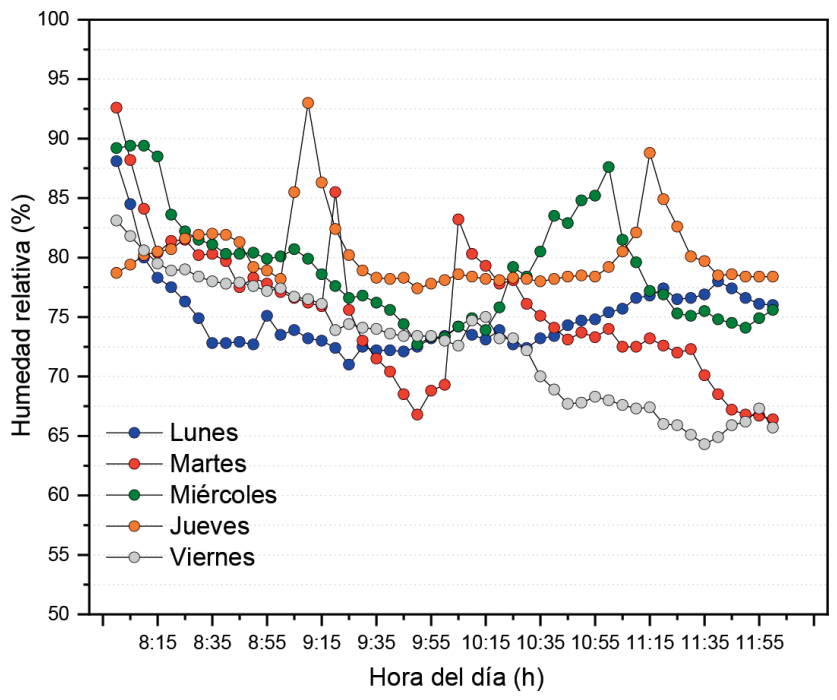

Figura C1. Variación de la humedad relativa

\section{Apéndice D}

(a)

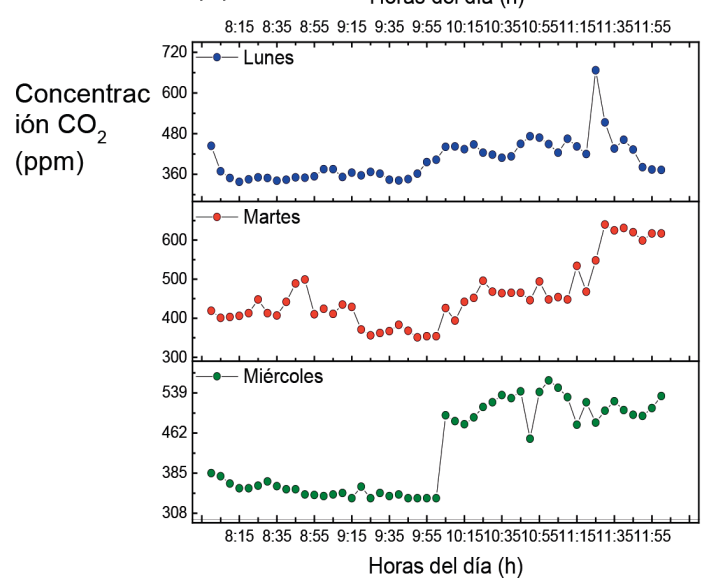

(b)

Horas del día (h)

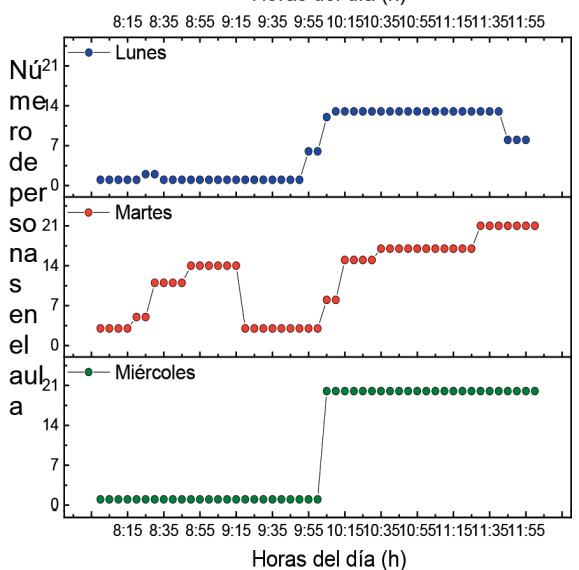

Figura D1. (a) Variación de la concentración de $\mathrm{CO}_{2}$ y (b) Ocupación del aula para los días lunes a miércoles 


\section{Apéndice $\mathrm{E}$}

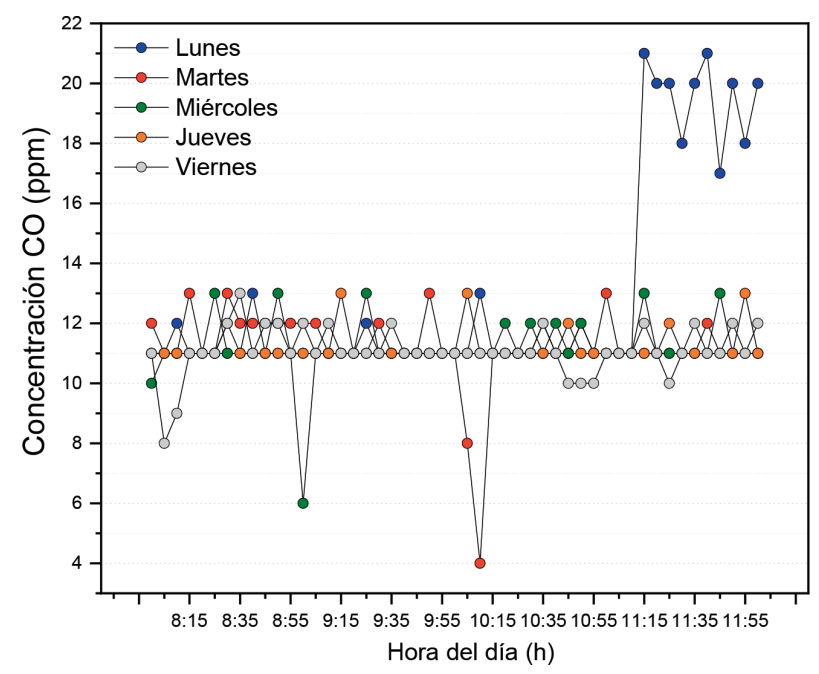

Figura E1. Variación de la concentración de CO

\section{Apéndice $F$}

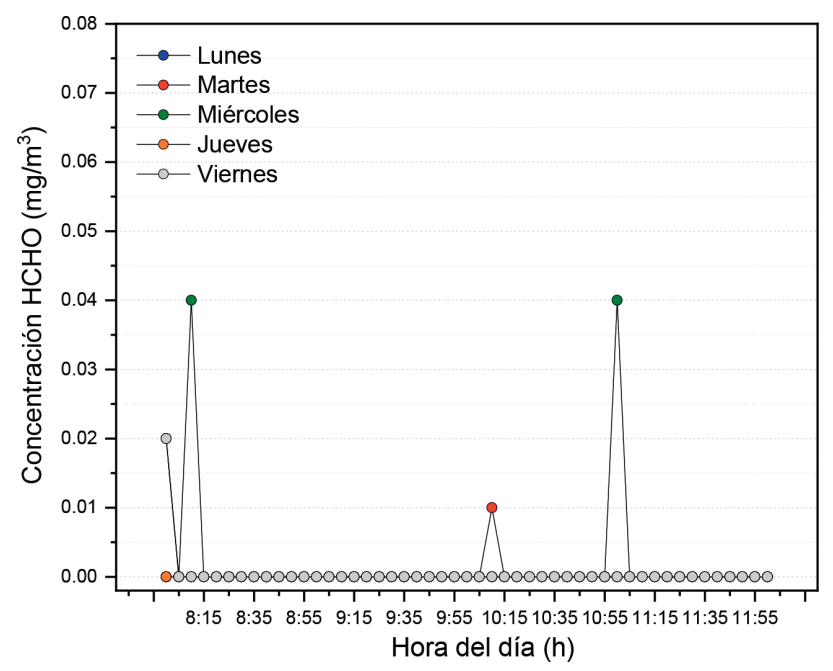

Figura F1. Variación de la concentración de HCHO 